\title{
Lexis and periods of literary history
}

\section{[Lexique et périodes de l'histoire littéraire]}

\author{
Michele Paolini
}

DOI: 10.18355/XL.2018.11.01XL.31

\begin{abstract}
Defining periods of literary history (not only in France) follows the facts that must be summarized. They do it on the basis of cultural conventions. "Nomina sunt consequentia rerum,» names are the consequence of things, and not vice versa. However, sometimes these lexical phenomena are more complex, depending on the different types of enunciative act. As this work begins to describe, in this lexical subsystem, on the one hand, neology plays an important role, and on the other, the national bases of the cognitive frameworks that are involved can never be ignored.

Key words: literary history, periodization, cognition, lexis
\end{abstract}

\section{Résumé}

Les termes qui définissent les périodes de l'histoire littéraire (pas seulement en France) suivent des faits qui doivent être résumés. Ils le font sur la base de conventions culturelles. "Nomina sunt consequentia rerum ", les noms sont la conséquence des choses, et non l'inverse. Cependant, ces phénomènes lexicaux sont parfois plus complexes, selon les différents types d'actes énonciatifs. Comme ce travail commence à décrire, dans ce sous-système lexical la néologie, d'une part, joue un rôle important, et les bases nationales des cadres cognitifs impliqués, d'autre part, ne peuvent jamais être ignorées.

Mots-clés: histoire littéraire, périodisation, cognition, lexique

\section{Introduction}

Chaque manuel destiné à l'enseignement de la littérature propose, à partir du sommaire, un corpus lexicale qui renvoie aux différentes périodes de l'histoire sur la base d'une double exigence de vulgarisation, spatio-temporel et sémantique (Polet, 2002, p. 733).

Voyons, par exemple, un livre en contexte FLE : Jean-Louis Boursin, Anthologie de la littérature française. Textes choisis du XIe au XXIe siècle, Paris : Belin, 2007.

En parcourant l'index (Boursin, 2007, p. 5), on peut voir cette liste de termes :

Le Moyen Âge ; La Renaissance ; Le Classicisme ; Les Lumières et la révolution ; Le "vague des passions"; Le Romantisme ; Esprit positif, esprit artiste ; La modernité en poésie ; Réalisme et Naturalisme ; Les Indépendants ; À l'aube du XXe siècle ; L'Entre-deux-guerres ; Sous l'Occupation ; Nouvelles voies ; Littératures francophones.

Les éléments (mots, concepts, syntagmes) qui définissent l'index constituent ici, comme on le voit, un ensemble tout à fait hétéroclite dans lequel certains d'entre eux relèvent de l'histoire, plutôt que de la littérature (Moyen Âge, L'Entre-deux-guerre), alors que d'autres expressions ont une fonction périodisante, bien qu'elles ne soient pas figées (Sous l'Occupation); et d'autres formes encore manquent, enfin, de tout contenu manifestement chronologique (Nouvelles voies, Littératures francophones). Un tel contenu d'ailleurs peut être indiqué implicitement à travers de véritables unités terminologiques (Renaissance ; Classicisme ; Romantisme ; etc.) qui dénomment les concepts propres du domaine historico-littéraire avec leur agencement cognitif. 
Dans ce contexte, le but de notre travail est de voir comment, sous quelles conditions et dans quelle mesure le lexique, avec les présupposés nationaux qu'il reflète parfois, répond à nos besoins épistémologiques dans le discours méta-littéraire. Et cela sur deux niveaux : dans une perspective (empirique) interculturelle et dans la perspective (potentielle) d'une "conscience littéraire supra-nationale", généralement souhaitable, en tout cas indispensable pour aborder la notion de littérature universelle (Weltliteratur, selon le terme inventé par Goethe en 1827) (Migliorini, 1977, p. 108), particulièrement recommandable dans le contexte européen.

\section{Lexique, littérature, Weltliteratur}

Bien qu'il soit composé d'éléments hétéroclites, cet ensemble de mots a quelque chose qui le rend solidaire : pas seulement la nature de signes linguistiques partagée par ses composants, mais surtout leur utilisation dans le discours sur la littérature. Ce qui n'est pas toujours, donc, le discours de la littérature (Todorov, 1987). En d'autres termes, cette partie du lexique, qui est méta-littéraire, est un sous-ensemble du lexique général, à l'innovation duquel elle contribue.

Par ailleurs, dans une perspective plus éloignée, le phénomène littéraire en tant que tel (textes primaires et textes secondaires) est un extraordinaire générateur de créations lexicales qui a des répercussions sur l'ensemble du vocabulaire. Dans ce cadre, les mots qui se produisent dans des contextes littéraires deviennent des clichés ou des unités de la langue commune, dans laquelle nous trouvons que certaines questions deviennent shakespeariennes, certains individus des dons Quichottes, certaines situations ubuesques, certaines idées baroques, certains appétits pantagruéliques, ou que certaines attitudes relèvent du bovarisme ou, encore, qu'un choix peut être cornélien et un endroit romantique. Les noms des auteurs, aussi bien que les mots qui désignent périodes et personnages littéraires, imprègnent profondément notre discursivité quotidienne. Et cette observation parfois doit être reprise même étymologiquement, car, par exemple, comme on le sait, le goupil a été désigné renart du fait de la redaction du Roman de Renart (XIIe siècle), devenu renard à partir du XVIe (Hamon, 1992, p. 106). Sans parler des nombreux cas détectables grâce à un inventaire de l'antonomase, figure de style qui nous permet de qualifier d'Harpagon un avare, de Tartuffe un hypocrite, de don Juan un séducteur, de Cassandre un (ou une) prophète de malheur, de Rastignac un ambitieux, cas souvent décrits et documentés par les dictionnaires. Ici, il serait très problématique d'établir une distinction claire entre le domaine linguistique et le domaine littéraire.

Néanmoins, l'utilisation de ces mots, en particulier ceux concernant les périodes, est bien loin d'être rigoureuse. Pire : elle est dépourvue de toute définition fiable même dans les contextes les plus "périodisés" : postes, programmes, livres (comme nous l'avons vu) et cours universitaires (Rohou, 2002, p. 707). D'ailleurs, sont toujours très appréciables, même pour leur ton, qui tend à relativiser, les mots que Jean Rohou a utilisés, lorsqu'il a remarqué que "peu de grands écrivains français ont eu le mauvais goût de s'asseoir sur deux siècles" (Rohou, 2002, p. 707 n 3). Et pourtant, ce phénomène - historiquement indésirable - a parfois eu lieu : Malherbe, Bayle, D’Aubigné, etc. n'ont pas vécu dans les limites imposées par une division séculaire. Ce qui rend toute catégorisation basée sur les siècles assez défectueuse. Il n'est que d'observer la désignation du "Grand Siècle", avec son implication d'un rôle central.

\section{Types d'actes énonciatifs}

Certaines distinctions préalables peuvent être utiles. Notamment, quand il est nécessaire de savoir si un terme, en ce qui concerne sa sémantique, doit être pris comme une sorte de dogme ou peut être considéré comme le point de départ d'un exercice sur différents points de vue possibles. La marge de manœuvre mise à la disposition du destinataire, pour qu'il coopère à la détermination du sens, s'inscrit 
dans un continuum entre un minimum, la monosémie, et un maximum représenté par la plus large polysémie. L'excursion le long de ce continuum est produite (il nous semble) par la nature même des actes énonciatifs. Nous y reviendrons plus tard. En tout cas, l'utilisation des termes doit être problématisée de façon critique, afin de ne pas se trouver dans une nuit lexicale où "tous les chats sont noirs", comme on dit, c'est-à-dire où toutes les positions les plus extravagantes peuvent être mises au même niveau que les positions méthodologiquement fondées, à cause d'une utilisation naïve et fallacieuse des mots.

Il y a trois types d'actes énonciatifs qui nous permettent (entre autres) de désigner une période littéraire : 1) on peut indiquer un concept de l'histoire littéraire avec un nom, quand le concept et le nom existent déjà. Nous définissons ce type comme désignation. 2) On peut imposer un nom à un concept, quand le concept existe déjà. Nous désignons alors ce type comme dénomination. 3) On peut créer un mot et le lier à un ensemble de propriétés sémantiques. Dans ce cas le concept et le mot existent à partir de l'acte énonciatif et par l'acte énonciatif. Nous désignons ce troisième type comme nomination. Les différents actes énonciatifs déterminent différents degrés de certitude (et marges de manœuvre) du point de vue du lecteur.

Les types numéro 2 et 3 ont un impact sur la phénoménologie de la création lexicale. Ce qui souligne dans quelle mesure notre sous-système est, comme on vient de le dire, néologique. Une telle néologie, d'ailleurs, est formelle, savante, souvent obtenue par composition (préfixes, suffixes, etc.). Et, quand elle établit le signifiant avec son sens, nous n'avons pas de place pour la pluralité des interprétations. Voyons, par exemple, le mot futurisme (http://www.cnrtl.fr/etymologie/) :

Étymol. et Hist. 1. 1909 « mouvement littéraire né en Italie qui se proposait de renouveler complètement les arts » (Le Figaro, 20 févr. loc. cit.); 2. 1917 « attitude d'esprit qui consiste à se tourner résolument vers le futur » (Loti, Vertige mond., p. 132). Empr. à l'ital. futurismo, attesté dep. 1909, date à laquelle F.-T. Marinetti fonda ce mouvement.

La nouveauté du mot n'a pas été produite dans l'anonymat de l'échange social (la langue saussurienne), car le futurisme nait en même temps, en tant que mouvement et en tant que marqueur lexical, créé par son promoteur Marinetti au moment de la naissance du groupe même. C'est un "mot d'auteur" (Migliorini, 1977 [1975]), dont la nouveauté a été produite par réalisation singulière (la parole saussurienne). Nomination est donc l'action de nommer, de désigner quelque chose par un nom, mais c'est aussi une structuration sémantique du mot. En ce qui concerne son utilisation à des fins de périodisation, futurisme représente en outre une indication lexicale claire, rapportée à un mouvement culturel. Une telle indication s'étend sur le plan sémantique - au sens figuré - à la période où il apparaît. Le futurisme peut être compris en tant que "période" tout comme le déroulement du travail d'un peintre tel que Pablo Picasso est normalement classé en "périodes", dont "la période cubiste" est particulièrement importante.

Toutefois, une distinction entre actes énonciatifs ne suffit pas, en elle-même, pour notre but. Nous pouvons encore essayer de mettre en pratique une classification par type de données. En effet, les informations auxquelles chaque mot renvoie peuvent être divisées en trois dimensions : une dimension cognitive, qui transmet l'information conceptuelle (le signifié) ; une dimension linguistique, qui transmet des informations sur le mot (le signifiant); une dimension communicative (ou pragmatique), qui transmet des informations sur l'utilisation (et sur les utilisateurs). Ce fil conducteur nous permet de voir que les observations sur les phénomènes néologiques s'appliquent à la dimension linguistique, alors que les informations portant sur les responsables des créations néologiques (les auteurs, les historiens) concernent la 
dimension pragmatique. Finalement, l'arrangement conceptuel et de catégorisation (esthétique, stylistique et chronologique) - impliqué dans la définition précise du mot - concerne la dimension cognitive.

\section{L'exemple du terme Moyen Âge}

Appliqué à la littérature, le nom composé Moyen Âge ne couvre que la deuxième partie de sa durée historique, qui va, selon l'une des conventions possibles, de la chute de l'Empire romain à la prise de Constantinople. En plus, Moyen Âge est l'un des trois termes historiques, avec l'antiquité et l'époque moderne, utilisés pour désigner et délimiter l'histoire de l'Europe, et, en l'occurrence, une période intermédiaire. Quant à l'âge, d'après le dictionnaire Lexis $(1979$, p. 40) :

\section{Âge. Epoque, durée determinée pendant laquelle une chose existe (...).}

Parmi les acceptions enregistrées par le dictionnaire Larousse en ligne (http://www.larousse.fr) :

Âge. Période de l'évolution du monde ou de l'humanité, de durée assez longue, considérée en fonction d'un fait significatif qui l'a marquée, d'ordre historique, technique, culturel, religieux, etc. : Âge du bronze, du fer. L'âge atomique.

Donc, la fonction spécifique du mot âge est de bien déterminer la sémantique particulière de la périodisation, sa condition gnoséologique de "durée déterminée", selon le Lexis, tandis que le mot moyen situe son sens dans un contexte stadial, progressif, préordonné et articulé. Un tel contexte est, en tout cas, complètement rétrospectif, car les auteurs "médiévaux" - Chrétien de Troyes, Guillaume de Lorris, Jean de Meung et tous les autres, ceux qui sont célèbres comme les anonymes n'avaient, quant à eux, aucun sentiment d'une telle intermédiarité historique. Ils considéraient tout simplement l'âge où ils vivaient comme le dernier avant la fin du monde. De surcroît, quand ils devaient définir cette période, ils la qualifiaient de nova, « moderne ». L'expression elle-même de media tempestas, puis de medium aevum, n'aurait paru que dans la toute dernière partie du Moyen Âge, pour être mise en place définitivement et acceptée d'une manière générale seulement au XVIIe siècle. Sous cette étiquette de Moyen Âge, évidemment, se rassemblent des différents phénomènes, processus et événements. Enfin, la valeur de ce label terminologique est principalement formelle.

Nous avons trouvé une approche interlinguistique et comparative, à ce sujet, intéressante. Notre analyse a été basée sur l'examen de quelques dictionnaires représentatifs des langues espagnole, française et italienne. Or, si nous consultons les dictionnaires français, que se passe-t-il ? D'après Le Grand Robert (Vol. 1, 2001, p. 245) :

Moyen Âge : époque qui va de la chute de l'Empire romain d'Occident, en 476, à la prise de Constantinople, en 1453.

Ce qui ne coïncide pas avec le contenu sémantique de la définition donnée à cet égard par le Lexis, déjà mentionné, selon lequel

Moyen Âge : période de l'histoire comprise entre le $V_{e} s$. (dislocation de l'Empire romain) et le milieu ou la fin $d u X V_{e}$ s. (chute de l'Empire byzantin ou découverte de l'Amérique ... ).

XLinguae, Volume 11 Issue 1XL, January 2018, ISSN 1337-8384, eISSN 2453-711X 
En fait, les termes de cette chronologie sont immédiatement remis en question : chute de l'Empire byzantin, c'est-à-dire 1453, ou découverte de l'Amérique, c'est-àdire 1492 ? Ce différentiel de 39 ans ne peut pas être considéré comme minuscule ou microscopique.

Regardons maintenant deux dictionnaires espagnols. Le dictionnaire de l'Académie royale espagnole (Diccionario de la lengua española, actualización 2017, http://dle.rae.es) propose cette définition :

\section{Edad Media : edad histórica que comprende desde el siglo V de la era cristiana hasta fines del siglo $X V$. \\ [Moyen Âge : âge historique qui comprend du siècle Ve de l'ère chrétienne jusqu'à la fin du siècle XVe].}

La définition donnée par le Diccionario del Español actual de Seco et Ramos (2008 [1999], Vol. 1, p. 1704) semble, quant à elle, complètement anodine : Edad Media apparaît seulement indirectement, à propos de Edad :

Edad : espacio amplio de tiempo, constituido por varios siglos, de aquellos en que se divide la historia y la prehistoria (...). - antigua, - media, moderna, - contemporanea (...).

[Âge : ample espace de temps, constitué de plusieurs siècles, de ceux où l'histoire et la préhistoire sont divisées (...). - ancien, - moyen, - moderne, - contemporain (...).]

Nous pouvons autrement chercher l'adjectif medieval (Diccionario del Español actual, 2008 [1999], Vol. 2, p. 3014) correspondant à médiéval :

Medieval : de la Edad Media.

[Médiéval : du Moyen Âge].

$\mathrm{Au}$ terme de ce parcours, un dictionnaire monolingue italien, le Dizionario della lingua italiana Devoto-Oli (1995, p. 1172) nous a semblé plus explicite, en nous rappelant les racines culturelles de la sémantique de ce mot :

Medioevo : età intermedia che la coscienza umanistica del Rinascimento individua tra il momento della propria comparsa e affermazione e la 'fine' del mondo antico (recuperato solo adesso nella sua autenticità tramite operazioni di tipo filosofico e archeologico): il termine ha in realtà un valore quasi esclusivamente culturale e solo convenzionalmente cronologico tra le due date 476 d.C. (Odoacre, re degli Eruli, deposto Romolo Augustolo, invia alla corte di Costantinopoli le insegne dell 'Impero Romano d'Occidente) e 1492 (sbarco di Cristoforo Colombo nel Nuovo Continente). [Moyen Age : âge intermédiaire que la conscience humaniste de la Renaissance identifie entre le moment de son apparition et de son affirmation et la « fin » du monde antique (récupéré seulement dans son authenticité par des opérations de type philosophique et archéologique) : le terme a une valeur presque exclusivement culturel et seulement conventionnellement chronologique entre les deux dates 476 apr. J.-C. (Odoacre, roi des Hérules, destitué Romulus Augustule, envoie les insignes de l'Empire romain d'Occident à la cour de Constantinople) et 1492 (débarquement de Christophe Colomb sur le Nouveau Continent).]

Un autre dictionnaire italien, grand et de grande envergure, le Grande dizionario della lingua italiana (GDLI, Vol. IX, 1975, p. 1039), clarifie le caractère relatif des 
déterminations chronologiques impliquées dans le mot :

Medioevo : periodo di transizione dall'evo antico all'evo moderno; secondo la convenzione più diffusa decorre dalla caduta dell ?Impero Romano d'Occidente nel 476 d.C. alla scoperta dell'America 1492.

[Moyen Âge : période de transition de l'ère antique à l'âge moderne ; selon la convention la plus répandue, il commence à partir de la chute de l'Empire romain occidental en $476 \mathrm{AD}$. à la découverte de l'Amérique 1492.]

En bref, nous avons un cadre chronologique produit à partir d'un ensemble de conventions qui peuvent varier selon les circonstances ou les critères d'approximation et de délimitation.

\section{Cadres chronologiques sur la base du lexique}

Variations chronologiques, interlinguistiques et diatopiques peuvent être basées sur le référent: différentes références géographiques doivent prendre en compte les phénomènes d'irradiation et de réception du référent. À ce sujet, bien que les référents géographiques semblent avoir un dénominateur lexical commun, les choses sont beaucoup moins simples. Par exemple, pour le Baroque, en tant que référent, le centre de rayonnement est l'Italie, les autres pays sont des récepteurs. Par conséquent, la correspondance des signifiants (Barroco en espagnol; Barocco italien; Baroque anglais ; Barock allemand, etc.) peut créer l'idée trompeuse d'un chevauchement parfait des signifiés au niveau interlinguistique, alors que ce mot, qui est polysémique même au niveau intralinguistique, au niveau interlinguistique peut refléter les différents moments de la diffusion du phénomène dans diverses parties de l'Europe. Donc, les différences interlinguistiques et diatopiques ont des conséquences dans l'agencement chronologique des cadres et indiquent parfois les temps d'irradiation du référent. Cela fonctionne différemment pour le mot Lumières (Enlightenment en anglais ; Illuminismo en italien ; Aufklärung en allemand ; Ilustración en espagnol) où les différences des signifiants n'induisent pas la même erreur ou malentendu sur le signifié. Dans ce cas, le problème des diffractions chronologiques possibles entre un centre rayonnant, la France, et les zones réceptrices reste.

Voyons, grâce à une ressource électronique (www.espacefrancais.com) quelques lexèmes, dont les références linguistiques et chronologiques reposent sur la base nationale française :

- Humanisme : 1530-1570

- Baroque : vers 1570-1650

- Classicisme : 1650-1700

- Romantisme : 1820-1850 (pour la France)

- Réalisme et Naturalisme : 1830-1890

- Mouvement dada : né en 1916

- Surréalisme : vers 1920-1940

Si nous revenons maintenant à notre exemple de texte, l'Anthologie de la littérature française (Boursin, 2007) nous notons que la comparaison montre certaines lacunes de corrélation. 


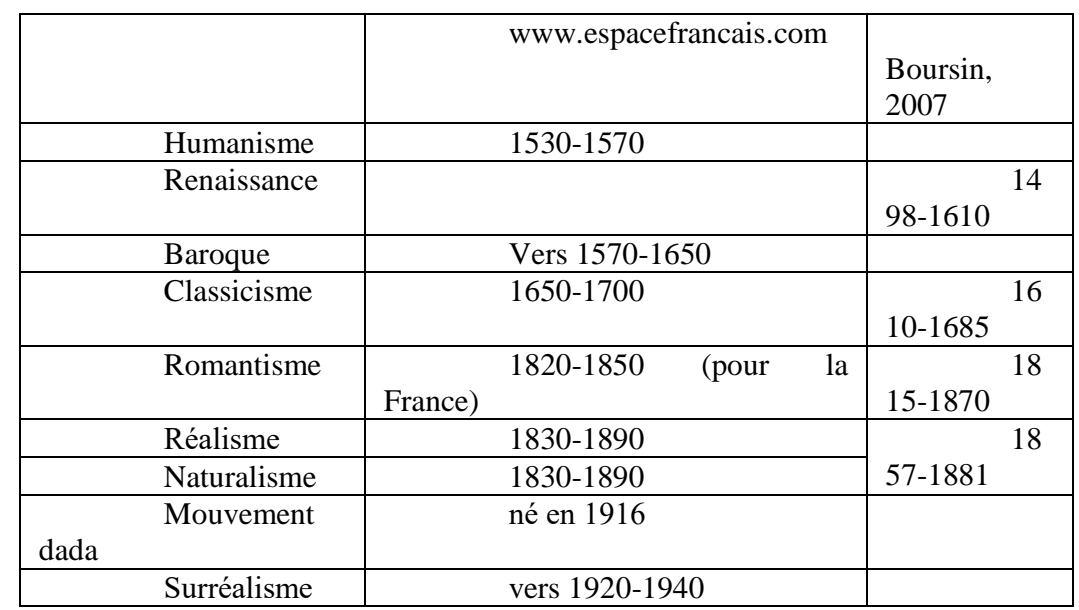

Tableau 1 : comparaison entre les différentes désignations chronologiques d'une ressource en ligne et un texte dans le contexte FLE

Nous voyons donc très clairement que la composition (et décomposition/recomposition) du contenu et les techniques de délimitation des cadres chronologiques correspondants ne sont pas traités de manière homogène.

\section{Resémantisations de mots préexistants}

Dans les deux cas de Renaissance et Baroque, les mots existent déjà, avec une signification commune non liée à l'histoire littéraire (renaissance 'nouvelle naissance', baroque 'de forme irrégulière') et sont récupérés avec une nouvelle spécialisation sémantique dans le contexte historico-littéraire. Nous avons donc des processus de transformation en termes de mots issus du lexique commun. Voilà ce que nous propose - dans toute sa complexité - la consultation d'un outil étymologique en ligne (http://www.cnrtl.fr/etymologie) :

Renaissance

Étymol. et Hist. 1. 1363 théol. renaiscence par baptesme (Miracles de N.D., XXI, 1456, éd. U. Robert, t. 3, p. 291); 2. av. 1563 « nouvelle naissance, réincarnation de l'âme après la mort » (La Boétie, Lettre de consolation de Plutarque à sa femme ds Cuvres, éd. P. Bonnefon, Bordeaux, 1892, p. 198); 3. a) 1598 en parlant d'un corps physique (Joub., Gr. chir., p. 430 ds Gdf. Compl. : Cheute des cheveux et renaissance de subtils); b) 1515 sylvic. (Ordonnance sur la chasse, les forêts, droits d'usage, in Rec. gén. anc. lois fr. t. 12, p. 60 ds Quem. $D D L$ t. 30); 4. fig. a) a) 1692 « réapparition nouvel essor (de quelque chose) » la renaissance des lettres humaines (Le Maistre ds Bouhours, Rem. nouv. sur la lang. fr., 3 éd., Paris, G. et L. Josse, p. 446); 1801 renaissance des sciences (Crèvecœur, Voyage, t. 1, p. 226); $\boldsymbol{\beta}$ ) spéc. 1828 hist. litt. poètes de la renaissance venus du temps de Henri II (Sainte-Beuve, Tabl. poés. fr., Append. pièces et notes), cf. id. cette grande renaissance des lettres (Id., ibid., p. 41); 1829 beaux-arts les artistes de la Renaissance (H. Fortoul ds R. de Paris, 1829, t. 3, p. 43, d'apr. M. Françon ds Mod. lang. Notes, t. 72, 1957, p. 200); 1831 (Balzac, Peau chagr., p. 20); en appos. avec valeur d'adj. 1835 maison renaissance (Michelet, Journal, p. 196); 1848 ameublement renaissance (Flaub., Champs et grèves, p. 159); b) 1816 « nouvelle naissance, épanouissement 
(de quelqu'un) » (Maine de Biran, Journal, 1814, p. 16). Dér. de renaître* d'apr. naissance*.

\begin{abstract}
Baroque
Étymol. ET HIST. I.- Adj. 1. 1531 joaill. se dit de perles de forme irrégulière (Inv. de Charles-Quint, $\mathrm{f}^{0} 786$ à 788 dans Gay, s.v. ajorffe : 97 Gros ajorffes dictz barroques enfillez en 7 filletz pes. ens. 1 onc. 12 grains); 2.1718 p. ext. fig. « bizarre, choquant » (Autreau, Port à l'Anglais, prologue, Euvres, éd. 1749, I, 6 dans Barb. Misc. 1, n ${ }^{0} 5$ : Il est vrai qu'il a l'accent un peu baroque), attesté dans les dict. dep. Ac. 1740; spéc. 1749 B.-A. d'un style architectural et décoratif qui s'écarte des règles de la Renaissance classique (Courajod, Livre-journal de Lazare Duvaux, marchand-bijoutier ordinaire du Roy [1748-58], 1873, t. 2, p. 19 : Une terrasse baroque en bronze doré d'or moulu, [...], pour une dormeuse, 175 1.).

Subst. p. ell. 1788 archit. " style architectural très orné et tourmenté » (Quatremère de Quincy, Encyclop. méthod., Archit., t. 1 cité par B. Migliorini, Manierismo, baròcco, rococò, Rome, 1962, p. 46 : Le baroque, en architecture, est une nuance du bizarre. Il en est, si on veut, le rafinement, ou, s'il étoit possible de le dire, l'abus [...] Barromini a donné les plus grands modèles de bizarrerie, Guarini peut passer pour le modèle du baroque) (...).
\end{abstract}

Quoi qu'il en soit, comme nous l'avons constaté, les démarcations chronologiques peuvent donc diverger en raison de différents problèmes : critères d'approximation à différentes échelles (le siècle, le demi-siècle, le quart de siècle, etc.), et dans ce cas il pourrait y avoir des décalages sémantiques intra-linguistiques (semantisations divergentes), mais aussi interlinguistiques, ou même - par ailleurs - des déviations interculturelles et référentielles. Sur ce dernier point, chaque fois qu'une guerre est identifiée, dans un certain contexte linguistique, comme référence chronologique, générale et par conséquent littéraire, nous devons toujours prendre en compte les différences possibles dans les histoires nationales. La "Grande Guerre », par exemple, ne désigne pas la période de 1914 à 1918 à l'unanimité, étant donné que pour les Italiens elle n'a commencé qu'en 1915, alors que pour les Russes elle s'est terminée en 1917. Cela a, bien évidemment, des répercussions, entre autres, sur le cadrage et sur la contextualisation des œuvres.

Pour ce qui est de la catégorisation, il est opportun de revenir à l'exemple du mot Baroque. En fait, à cet égard, nous devons nous confronter à une possible ambivalence sémantique. Le Baroque est-il un style ou une période ? Si nous revenons à la comparaison entre les trois grandes langues romanes, voici quelques observations lexicographiques.

Baroque : style (Le Grand Robert, Tome 1, 2001, p. 1231).

Barroco : estilo arquitectónico (...), literario (...), musical (Diccionario de la lengua española, http://dle.rae.es).

[Baroque : style architectural (...), littéraire (...), musical].

Barocco : stile secentesco (GDLI, Vol. II, 1962, p. 76).

[Baroque : style du XVIIe siècle].

Ici l'information chronologique est intrinsèque au genus proximum seulement dans un cas, c'est-à-dire le dernier : « style du XVIIe siècle ».

XLinguae, Volume 11 Issue 1XL, January 2018, ISSN 1337-8384, eISSN 2453-711X 
Le mécanisme métonymique qui déplace, par extension, le champ sémantique du style à sa chronologie relative, est bien illustré par le dictionnaire italien Devoto-Oli (1997, p. 206) :

Barocco : il periodo storico-culturale successivo alla Controriforma e nei paesi cattolici, caratterizzato da un'arte affollata di virtuosismi e di vistosità, programmaticamente disancorata dai canoni rinascimentali, spesso, ma a torto, ritenuta povera di contenuto spirituale.

[Baroque : la période historico-culturelle qui a suivi la Contre-Réforme et dans les pays catholiques, caractérisée par un art plein de virtuosité et de perceptibilité facile, non amarrée par les canons de la Renaissance, souvent, mais à tort, réputée dépourvue de contenu spirituel].

\section{Conclusion}

L'application, dans les contextes éducatifs, à tous les niveaux, du concept de Weltliteratur, à savoir de littérature mondiale, et les différents projets de construction d'un discours historico-littéraire supranational nécessitent des clarifications adéquates et des généralisations interculturelles qui doivent toujours être évaluées sur la base d'une approche comparative.

Comme nous avons pu le constater, dans la terminologie des périodes littéraires il n'est pas nécessairement dit que les mots suivent les « choses », c'est-à-dire, dans ce cas-ci, les cadres cognitifs qui contiennent des informations de base sur les tendances littéraires, leurs esthétiques et leurs périodes. Parfois, bien au contraire, «res sunt consequentia nominum », les " choses » sont une conséquence des mots. Nous l'avons vu à travers l'exemple des néologismes formés par le suffixe -isme, tels que futurisme, surréalisme, etc. La force expressive et productive, mais aussi les ambiguïtés cachées dans la sémantique du suffixe -isme, sont également démontrées par certaines répercussions dues à sa lexicalisation. Selon le dictionnaire Larousse en ligne le mot isme, dont la valeur serait péjorative, n'est qu' indirectement rattaché au contexte littéraire :

Isme : courant politique, religieux, philosophique, etc., qui, par une dérive doctrinaire, peut devenir une menace pour la liberté.

La définition de l'équivalent espagnol ismo, donnée par le Diccionario de la lengua española DRAE (http://dle.rae.es), est bien différente, dans la mesure où elle se réfère, sans aucune sorte de médiation, à des phénomènes littéraires et artistiques :

Ismo : movimiento artístico, literario o filosófico. (...) Referido a movimientos efimeros, como los que integraron el vanguardismo.

[Isme : mouvement artistique, littéraire ou philosophique. (...) Se référant à des mouvements éphémères, comme ceux qui forment l'avant-garde].

Ces ismes, nous l'avons vu, ne suivent pas toujours les voies d'une sémantique qui les précède. Au contraire, ils coïncident quelquefois avec sa création et sa structuration. Quant aux ismi de la tradition linguistique italienne, eux aussi se réfèrent plutôt clairement, et sans intermédiaires, à l'activité littéraire en tant que telle. En témoigne clairement l'essai que Luigi Capuana avait intitulé, d'une manière emblématique, avant même l'aube du XXe siècle, en 1898, Gli "ismi » contemporanei (Les « ismes » contemporains). Par le biais de ce titre, le discours se plongeait dans le débat littéraire et critique de son époque d'une manière audacieuse et perspicace. Le nouveau mot italien, les ismi - les ismes de la langue française et les ismos de la langue espagnole - dégageait alors la force de son originalité : celle de résumer une époque, et en même temps de la problématiser. 


\section{Bibliographic references}

BOURSIN, J.-L. 2007. Anthologie de la littérature française. Textes choisis du XIe au XXIe siècle. Paris : Belin.

CABRÉ, M. T. 1998. La terminologie : théorie, méthode et applications. Les Presses de l'Université d'Ottawa, Armand Colin.

CAPUANA, L. 1898. Gli 'ismi' contemporanei. (Verismo, Simbolismo, Idealismo, Cosmopolitismo). Catania : Giannotta.

GREIMAS, A.J. - COURTÉS, J. 1993. Sémiotique. Dictionnaire raisonné de la théorie du langage. Paris : Hachette.

GUILBERT, L. 1973. La spécificité du terme scientifique et technique. In : Langue française, n. 17, pp. 5-17.

HAMON, A. 1992. Les mots du français. Paris : Hachette.

HANDWERKER, B. 1989. Savoir lexical, savoir expert: problèmes de représentation. In : DRLAV, Revue de linguistique, n. 40, pp. 63-87.

MIGLIORINI, B. 1977 [1975]. Parole d'autore. Onomaturgia. Firenze : Sansoni.

MORTUREUX, M.-F. 1984. La dénomination, approche socio-linguistique. In : Langages, $19^{\mathrm{e}}$ année, n. 76, pp. 95-112.

NIKLAS-SALMINEN, A. 2015 [1997]. La lexicologie. Paris : Armand Colin.

PAOLINI, M. 2015. La natura del segno terminologico. In: Philologia, vol. XXV, n. 1, pp. 29-34.

POLET, J.-C. 2002. Périodisation et grands ensembles littéraires. Limites nationales et cohérences transversales. In: Revue d'histoire littéraire de la France, vol. 102, 2002/5, pp. 733-746.

QUEMADA, B, 2006. Problématiques de la néologie. In : Che fine fanno i neologismi ?, G. Adamo - V. Della Valle (eds.). Firenze : Olschki, pp. 1-21.

ROHOU, J. 2002. La périodisation : une reconstruction révélatrice et explicatrice. In : Revue d'histoire littéraire de la France, vol. 102, 2002/5, pp. 707-732.

TODOROV, T. 1997. La notion de littérature. Paris : Éditions du Seuil.

\section{Dictionnaries}

Diccionario del Español actual 2008 [1999]. M. Seco - G. Ramos (eds.). Madrid : Santillana.

Grande Dizionario della Lingua Italiana (GDLI) 1961-2002. S. Battaglia (ed.). Torino : Utet.

Il Dizionario della lingua italiana 1995. G. Devoto - G. C. Oli (eds.). Firenze : Le Monnier.

Le Grand Robert de la langue française, deuxième éd. 2001. Paris : Dictionnaires Le Robert.

Lexis Larousse de la langue française1979. Paris : Larousse.

\section{Sources Internet}

EspaceFrancais.com. In : http:// www.espacefrancais.com [consulté le 20/10/2017].

Etymologie - Centre National de Ressources Textuelles et Lexicales. In : http://www.cnrtl.fr/etymologie/ [consulté le 20/10/2017].

Words: 4793

Characters: 31603 (17,55 standard pages)

Dr. Michele Paolini, PhD.

Department of Romance Languages and Literature

Faculty of Education

Comenius University in Bratislava

Račianska 59, 81334 Bratislava

Slovakia

paolini@fedu.uniba.sk

XLinguae, Volume 11 Issue 1XL, January 2018, ISSN 1337-8384, eISSN 2453-711X 